\title{
Automatic Face Recognition for
} Forensic Identification of Persons Deceased in Humanitarian Emergencies

\author{
Ruggero Donida Labati \\ Department of Computer Science \\ Università degli Studi di Milano \\ Milano, Italy \\ ruggero.donida@unimi.it \\ Barbara Bertoglio \\ Department of Biomedical Sciences for Health \\ Università degli Studi di Milano \\ Milano, Italy \\ barbara.bertoglio@unimi.it \\ Fabio Scotti \\ Department of Computer Science \\ Università degli Studi di Milano \\ Milano, Italy \\ fabio.scotti@unimi.it
}

\author{
Danilo De Angelis \\ Department of Biomedical Sciences for Health \\ Università degli Studi di Milano \\ Milano, Italy \\ danilo.deangelis@unimi.it \\ Cristina Cattaneo \\ Department of Biomedical Sciences for Health \\ Università degli Studi di Milano \\ Milano, Italy \\ cristina.cattaneo@unimi.it \\ Vincenzo Piuri \\ Department of Computer Science \\ Università degli Studi di Milano \\ Milano, Italy \\ vincenzo.piuri@unimi.it
}

\begin{abstract}
Forensic scientists often need to identify deceased people. The identification process mainly consists of the analysis of the DNA, dental records, and physical appearance. In humanitarian emergencies, the antemortem documentation needed for forensic analyses may be limited. In this context, face recognition plays a relevant role since antemortem pictures of missing persons are commonly made available by their families. Therefore, automatic recognition systems could be of paramount importance for reducing the search time in databases of face images and for providing a second opinion to the scientists. However, there are only few preliminary studies on automatic face recognition methods for forensic applications, and none of the works in the literature consider problems related to humanitarian emergencies. In this paper, we propose the first study on automatic face recognition for humanitarian emergencies. Specifically, we propose a recognition methodology and we analyze the accuracy of different biometric methods based on deep learning strategies for a real study case. In particular, the considered data regard recent deaths of migrants in Mediterranean Sea. The obtained results are satisfactory, and suggest that automatic recognition methods based on deep learning strategies could be effectively adopted as support tools for forensic identification.
\end{abstract}

Index Terms-Biometrics, Forensics, Face recognition, Deceased, Deep learning

This work was supported in part by the EC within the H2020 Program under projects MOSAICrOWN and MARSAL, by the Italian Ministry of Research within the PRIN program under project HOPE, and by the Università degli Studi di Milano under project AI4FAO. We thank the NVIDIA Corporation for the GPU donated.

\section{INTRODUCTION}

Forensic investigation aims at finding evidences to solve a crime. A challenging problem often faced off by forensic scientists consists of identifying deceased people. The identification process is mainly performed by analyzing the DNA, dental records, and physical appearance. The DNA analysis is highly accurate but is not feasible in many real contexts since it can be difficult to obtain sufficient antemortem data to be compared with that of the deceased [1]. Similarly, the evaluation of dental records can obtain accurate results, but antemortem clinical records are not always available. The analysis of physical characteristics is another relevant approach since it is generally easier to obtain antemortem pictures and descriptions of the deceased with respect to DNA and dental data. Examples of evaluated physical characteristics are the fingerprints, face, and soft biometric features like age, height, weight, biological sex, and race. From the physical characteristics, the face is the most relevant one since antemortem pictures of missing persons are commonly made available by their families.

In humanitarian emergencies, face recognition is particularly relevant. As an example, the analysis of face images permitted to recognize around one-third of the Thailand tsunami victims and around half of the victims in the ferry accident in Estonia in 1994 [2]. Furthermore, face recognition can be a preliminary screening for fastening the forensic identification of relevant numbers of people. In addition, it is possible to infer additional soft biometric information from the face, as 
the gender and age [3]. However, the recognition is usually performed by human operators and is not always accurate. For example, 18 victims of the Bali bombings in 2002 were wrongly visually identified by their relatives [2].

In the literature, there are only few preliminary studies on automatic face recognition for postmortem identification. There is a study on the decomposition effect on the accuracy of face recognition techniques [4], which considers face images acquired from bodies conserved in controlled environments and not presenting injuries. To the best of our knowledge, there is only a study on the recognition of injured faces, but it considers images acquired from living people [5]. To the best of our knowledge, there are no studies on automatic face recognition methods considering postmortem images affected by strong injuries and uncontrolled decomposition.

This paper presents the first preliminary study on automatic face recognition for forensic applications in humanitarian emergencies. The novelty is twofold. First, we present a methodology for automatic face recognition for forensic applications, which can adopt different techniques based on deep learning strategies. Second, we measure the accuracy of stateof-the-art deep learning strategies to evaluate the feasibility of automated face recognition techniques for forensic applications in humanitarian emergencies.

As study case, we consider the identification of victims of the Mediterranean sea crossings in the past years [6]. This context is particularly challenging for face recognition methods due to the low quality of postmortem and antemortem samples. Postmortem images can present relevant injuries and high level of decomposition caused by the seawater. Antemortem images can be old, present low resolution, and other severe non-idealities.

We evaluated the accuracy of the proposed automatic face recognition methodology using a dataset composed of 330 postmortem images and 218 antemortem images. The obtained results are satisfactory and prove that automatic face recognition methods could become useful tools for forensic scientists.

This paper is structured as follows. Section II describes the motivations for which the postmortem identification presents more challenges with respect to the recognition of living individuals. Section III summarizes the related works. Section IV presents the proposed methodology. Section V introduces the humanitarian emergency considered as study case. Section VI summarizes the performed experiments and the achieved results. Section VII concludes the work.

\section{Motivation}

Postmortem and antemortem samples present important differences and heterogeneous non-idealities. In fact, postmortem face images have usually been acquired in the place in which the deceased has been found or in the operating theater. Differently, the antemortem pictures typically available for humanitarian emergencies have usually been acquired in uncontrolled and uncooperative conditions.

The main non-idealities of postmortem samples for automatic face recognition systems are reported in the following.
- Eyes: the eyes are frequently closed and the irises are often decomposed.

- Injuries: the face images can present serious injuries, which caused the death of the individual.

- Deformations: the external environment can decompose the face and modify its shape. As an example the seawater can dilate the tissues of the face.

- Expression: the face expression is non-natural.

- Occlusions: the images can be affected by different occlusions, such as blood, froth, sand, dirt, or grass.

- Pose: the head can be placed with different rotations in the three-dimensional space.

- Light: especially for images acquired in the place in which the deceased has been found, the illumination can be uncontrolled and negatively affect the accuracy of face recognition systems.

The main non-idealities of antemortem samples for automatic face recognition systems are reported in the following.

- Time difference: the images could have been acquired ages before the death of the individual.

- Resolution: the images are frequently sent by email to the forensic scientists and typically present low resolution. Furthermore, the pictures frequently represent the full body of a group of person, thus presenting a limited number of pixels usable for face recognition.

- Artifacts: the images are frequently affected by artifacts introduced by digital scanners.

- Pose: the pose is uncontrolled and can be non-frontal with respect to the camera.

- Expression: the face expressions are uncontrolled.

- Occlusions: the face can be occluded by glasses, bear, hat, or makeup.

- Illumination: the illumination is uncontrolled and unknown.

Current face detection and recognition systems based on deep learning strategies have not been designed for this challenging context. Therefore, we present a preliminary study on the applicability of deep learning approaches for forensic face identification in humanitarian emergencies.

\section{RELATED WORKS}

In the literature, there are some studies on automatic biometric recognition techniques for forensic identification, which consider different traits. Most of the works are based on the fingerprints, which can achieve relevant identification accuracy for postmortem identification [7]. However, fingerprint recognition methods cannot be applied in a wide number of cases because of the unavailability of antemortem samples or because the finger skin of the deceased is degraded. Some studies evaluated the feasibility of postmortem iris recognition [8], revealing that the this biometric trait degrades in less than one week, even if the body is conserved in controlled environmental conditions.

There are only few studies on forensic applications of face recognition techniques. The study [9] focused on living 
individuals, and proposed techniques to fuse the decisions performed by automatic recognition methods and human experts. Another study [4] analyzed the effect of different postmortem degradation levels on the face recognition accuracy. Specifically, the authors analyzed the performance of some free and commercial off-the-shelf libraries for samples acquired at different time from the death. The obtained results are satisfactory, but this work only considered bodies conserved in controlled environmental conditions and not presenting injuries. There is only a study on biometric recognition of injured faces, but it focuses on living individuals [5]. Other works focus on matching skulls with face images [10].

To the best of our knowledge, we present the first study on face recognition for forensic identification of people deceased in humanitarian emergencies. Since state-of-the-art face recognition systems are based on deep neural networks composed of high numbers of layers and trained using big databases of samples acquired in uncontrolled conditions [11]-[13], we based our study on publicly available implementations of deep neural networks able to achieve highly accurate results for the recognition of living individuals.

\section{Proposed Methodology}

In the following, we propose a methodology to perform forensic identifications of deceased persons from face images. We consider the forensic identification of deceased persons as an open-set identification problem [14], in which one or more antemortem images are compared with a gallery database of postmortem samples. This process is based on biometric recognition techniques designed for identity verification and able to compute a matching score (similarity or dissimilarity measure) by comparing two samples. A decision threshold allows the system to determine if there is an identity in the gallery database that corresponds to the probe antemortem sample. If this test obtains a positive result, the system returns the identity corresponding to the gallery sample corresponding the best matching score.

The face recognition process is composed of three main steps: preprocessing, feature extraction, and matching. The proposed methodology presents differences in processing antemortem and postmortem face images. Fig. 1 shows the schema of the proposed methodology.

\section{A. Preprocessing}

The preprocessing step consists of detecting the face region and reducing eventual face rotations. For antemortem images, these tasks can be accurately performed by using state-ofthe-art techniques based on deep neural networks trained for samples acquired in uncontrolled conditions [15].

For postmortem images, we propose a human-driven preprocessing. First, a human operator is asked to rotate the postmortem image to align the face with the vertical axis. Second, the preprocessing software is executed. In the case in which the face region has wrongly been detected, a human operator is asked to crop the input image for extracting the bounding box the face region. This strategy aims at improving
Enrollment of a deceased

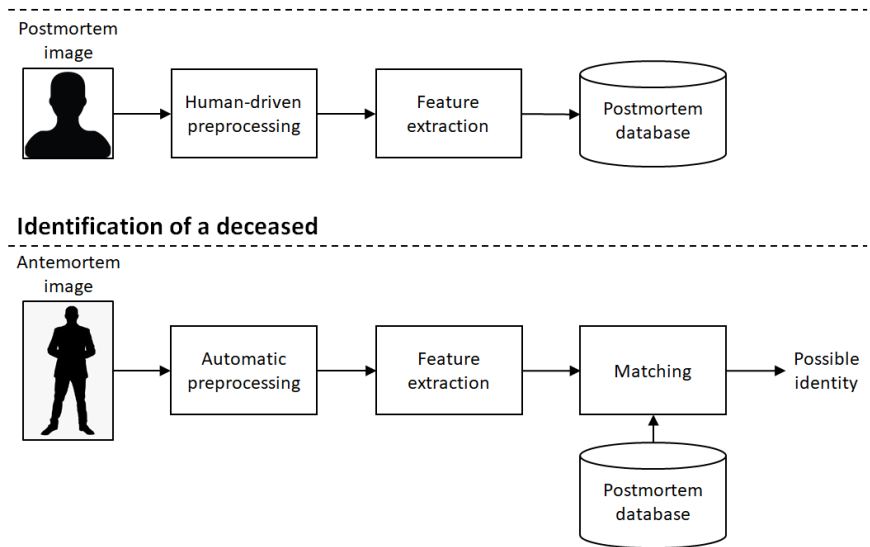

Fig. 1. Schema of the proposed face recognition methodology for the forensic identification of deceased people. In the enrollment step, one or more templates are extracted from face images and stored in the postmortem database. The postmortem identification step consists of an open set identification problem.

the unsatisfactory accuracy of state-of-the-art face detection techniques for postmortem images.

\section{B. Feature extraction}

The feature extraction step can be performed using different methods based on algorithmic approaches and deep neural networks [16]. To achieve state-of-the-art accuracy, we use three deep neural networks trained using big datasets of images collected from the web. Every selected network has an input layer accepting images of size $224 \times 224$ pixels. The size of the cropped face image is normalized accordingly.

The selected deep neural networks are VGG-16 [16], Resnet-50 [17], and SEnet [18]. The considered neural networks have been trained or fine-tuned using the following image datasets: VGGFace [16] (about 982 thousand images), MS-Celeb-1M [19] (about 10 million images), and VGGFace2 [20] (about 3.31 million images).

To compute the template, a feature vector is extracted from the layer of the network preceeding the fully connected layers. This feature vector is then L2-normed.

More details on the computational architectures are provided in the following.

- VGG-16: this deep neural network consists of the model presented in [21]. The network has been trained using the triplep loss approach [22] for VGGFace. The architecure is composed of 11 blocks, each consisting of a linear operator followed by one or more non-linear operators, such as ReLU and max pooling. The first 8 blocks are convolutional, while the last 3 blocks are fully connected.

- Resnet-50: the deep neural network consists of the model presented in [17]. The network has been trained for classification using the soft-max loss function for MSCeleb-1M, and fine-tuned for VGGFace2. The architecure is composed of 50 blocks, each consisting of a linear operator followed by one or more non-linear operators, 
such as ReLU and max pooling. The first 47 blocks are convolutional, while the last 3 blocks are fully connected.

- SEnet: the deep neural network consists of the model called SE-ResNet-50 [18]. The network has been trained for classification using the soft-max loss function for MSCeleb-1M, and fine-tuned for VGGFace2. The architecure is based on Resnet-50, but it includes Squeeze-andExcitation blocks [18], which adaptively recalibrate channelwise feature responses by explicitly modelling channel relationships. The first 47 blocks are convolutional, while the last 3 blocks are fully connected.

\section{Matching}

The matching score $s_{(A, B)}$ consists of the cosine distance between two templates $A$ and $B$, which is calculated as follows:

$$
s_{(A, B)}=\cos \theta=\frac{A \cdot B}{\|A\|\|B\|}=\frac{\sum_{i=1}^{n} A_{i} B_{i}}{\sqrt{\sum_{i=1}^{n} A_{i}^{2}} \sqrt{\sum_{i=1}^{n} B_{i}^{2}}},
$$

where $n$ is the number of elements composing each template.

\section{Study Case}

We analyzed real data related to a humanitarian emergency, collected by the forensic scientists of the LABANOF laboratory, Univeristà degli Studi di Milano, Italy. Specifically, we considered migrants dead in the Mediterranean Sea.

Every year, thousands of migrants die attempting to cross the Mediterranean Sea to reach the Italian coasts. More than $60 \%$ of these victims is still unidentified [6]. One of the main difficulties in their identification consists of the lack of antemortem data with sufficient quality for an accurate forensic analysis. In most of the cases, the available data are only photos and videos of poor quality. Furthermore, DNA analyses may at times present difficulties due to the lack of genetic information on the populations to which the victims belonged [1]. Therefore, automatic face recognition systems for postmortem identification could become extremely useful tools for the forensic investigations concerning this humanitarian emergency.

Most of the victims are from African countries. They can be males or females of different ages, including kids and infants.

\section{EXPERIMENTAL RESUlts}

This section describes the experimental dataset and presents the results achieved for the preprocessing step and for identity verification.

\section{A. Dataset}

To create the experimental dataset, we selected only the images with frontal pose from a set of samples collected by the forensic scientists. The dataset is composed of 330 postmortem images from 174 adult individuals (males and females) and 218 antemortem images from 47 adult individuals (males and females). There are 14 corresponding identities between postmortem and antemortem samples. Table II resumes the number of samples in the postmortem and antemortem sets.
TABLE I

NUMBER OF SAMPLES PER INDIVIDUAL FOR THE EXPERIMENTAL DATASET

\begin{tabular}{lcc}
\hline \hline & Postmortem & Antemortem \\
\hline Mean & 1.90 & 5.44 \\
Median & 2 & 4 \\
Minimum & 1 & 1 \\
Maximum & 8 & 31 \\
Standard deviation & 1.17 & 5.17 \\
\hline \hline
\end{tabular}

TABLE II

SIZE OF THE IMAGES OBTAINED COMPUTED BY THE PREPROCESSING STEP

\begin{tabular}{l|cc|cc}
\hline \hline & \multicolumn{2}{|c|}{ Postmortem } & \multicolumn{2}{c}{ Antemortem } \\
& $\begin{array}{l}\text { Height } \\
\text { (pixels) }\end{array}$ & $\begin{array}{c}\text { Width } \\
\text { (pixels) }\end{array}$ & $\begin{array}{c}\text { Height } \\
\text { (pixels) }\end{array}$ & $\begin{array}{c}\text { Width } \\
\text { (pixels) }\end{array}$ \\
\hline Mean & 1924.2 & 1596.6 & 300.3 & 250.2 \\
Median & 1991 & 1625 & 208 & 181 \\
Min & 300 & 257 & 26 & 23 \\
Max & 4222 & 3514 & 1537 & 1221 \\
Standard deviation & 945.7 & 766.3 & 260.2 & 213.1 \\
\hline \hline
\end{tabular}

The resolution of antemortem images is much lower with respect to that of postmortem images. Table II describes the size of the cropped face images computed by the preprocessing step.

\section{B. Preprocessing}

We evaluated the performance of different face detectors, and the deep neural network presented in [23] achieved the best results for both the antemortem and postmortem images. This face detector properly found the region of interest for $100 \%$ of the antemortem images. However, it failed in detecting the face for $21.5 \%$ of the postmortem samples. The regions of interest of the postmortem images wrongly detected by the automatic method have been manually marked by a human operator.

\section{Identity verification accuracy}

We evaluated the performance of different deep neural networks for identity verification. As figures of merit, we considered the Equal Error Rate (EER) [24] and Receiver Operating Characteristic (ROC) curves [25]. We performed three tests to analyze the face recognition in different conditions.

- PM: we considered only the set of postmortem samples, performing 534 genuine and 108036 impostor comparisons. The goal of this test is to evaluate the capability of processing postmortem images using deep neural networks.

- AM: we considered only the set of antemortem samples, performing 1704 genuine and 32336 impostor comparisons. The goal of this test is to evaluate the effect of the severe non-idealities characterizing the available antemortem images.

- AM-vs-PM: we considered the antemortem samples as probes and the postmortem samples as the gallery, performing 118 genuine and 60932 impostor comparisons. 
The goal of this test is to simulate a real forensic application, in which antemortem images are compared to the dataset of postmortem images for searching possible correspondences.

Fig. 2 and Table III summarize the results achieved by three deep neural networks (VGG-18, SENet, and Resnet-50) for the three performed tests (PM, AM, AM-vs-PM).

The results achieved for the test PM prove that deep neural networks can effectively process face images of deceased persons. In particular, Resnet-50 achieved the best recognition accuracy, with EER of $1.50 \%$. This result is particularly satisfactory, but it should be considered that the samples present less interclass variability with respect to the ones acquired from living individuals.

The results achieved for the test AM prove that the samples present severe non-idealities. In fact, SENet obtained the best accuracy, with EER of $10.36 \%$. The obtained EER is much higher with respect to the one reported for other big datasets designed to train and test face recognition systems for samples acquired in uncontrolled conditions [20]. Most of the false non matches have been obtained for low resolution images.

The results achieved for the scenario AM-vs-PM proved that deep neural networks could be useful tools for the forensic identification of deceased individuals. SENet obtained the best performance, with EER of $13.21 \%$. Compared to the results achieved for the test AM, this result can be considered as particularly positive. Furthermore, human operators achieved around $80 \%$ of accuracy in performing postmortem identifications in a similar scenario [2]. Most of the false non matches have been obtained for postmortem images presenting relevant injuries and deformations due to a long permance in the water.

\section{CONCLUSION}

This paper presented the first study in the literature on face recognition systems based on recent deep neural networks for the forensic identification of people deceased in humanitarian emergencies. The proposed methodology aims at identifying persons by comparing postmortem face images with antemortem samples.

We considered data related to a real humanitarian emergency, analyzing images of migrants dead in the Mediterranean Sea. Specifically, we used a dataset composed of 330 postmortem images and 218 antemortem images. The best performing deep neural network achieved EER of $13.21 \%$, which is better or comparable to the results obtained by human operators in a similar scenario. The obtained accuracy can therefore be considered as satisfactory, proving that automatic face recognition systems based on deep neural networks could become useful tools for forensic scientists.

Further studies will focus on deep learning approaches specifically designed for forensic images and on methods to improve the quality of both antemortem and postmortem images. To this purpose, we will cooperate with national and international institutes for collecting novel and bigger datasets of forensic images.

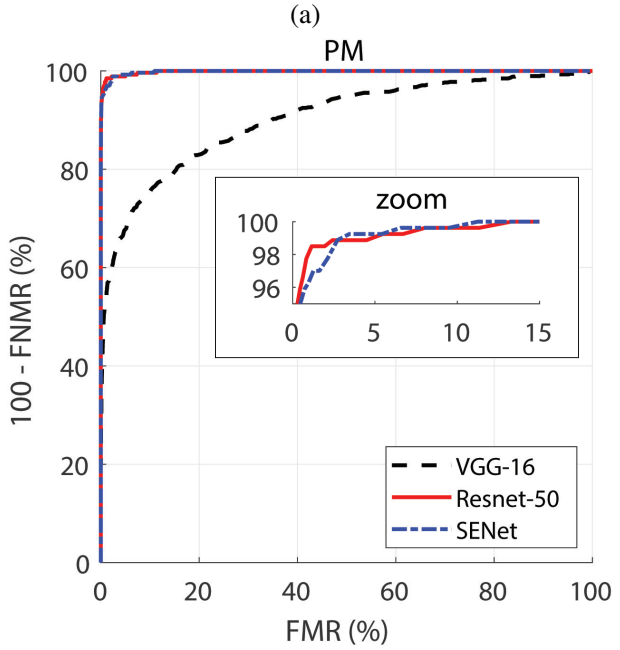

(b)
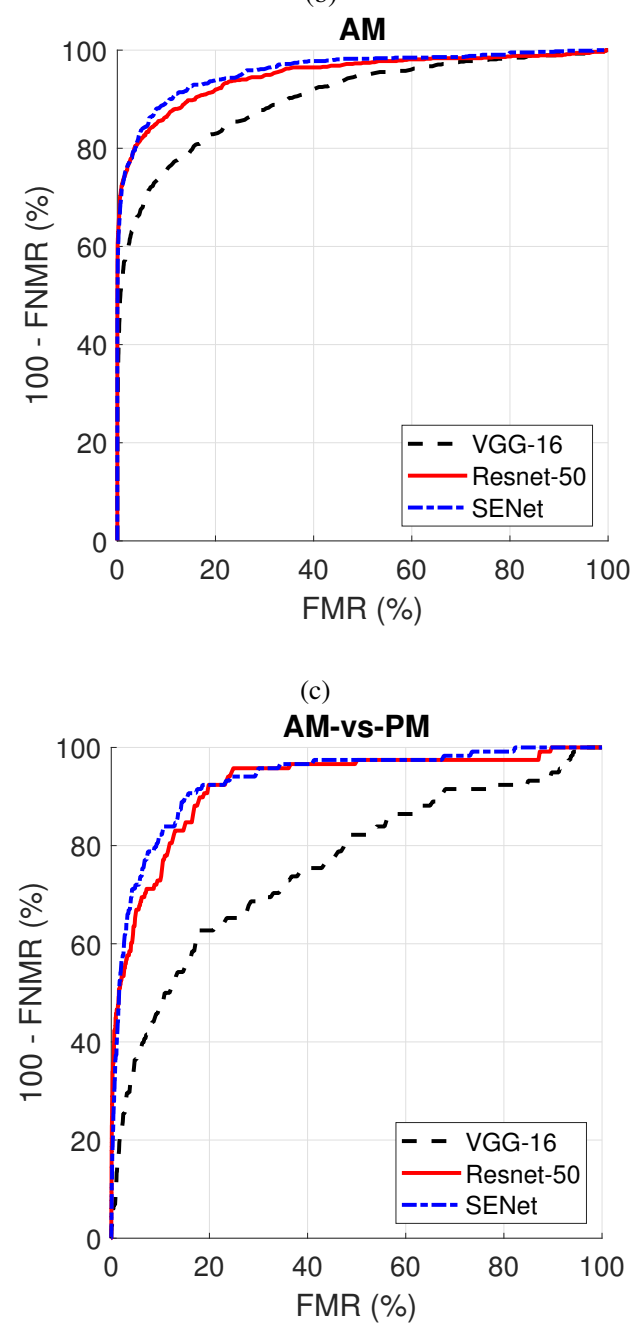

Fig. 2. ROC curves obtained by three deep neural networks (VGG-18, SENet, Resnet-50) for the scenarios: a) PM, b) AM, c) AM-vs-PM. The neural networks obtained satisfactory performance for postmortem images (PM). The results achieved for antemortem images (AM) show that the accuracy of the evaluated recognition methods is reduced by the non-idealities affecting the samples. The results achieved comparing antemortem and postmortem images (AM-vs-PM) prove that the best performing deep neural networks could represent useful tools for the forensic identification of deceased persons. 
TABLE III

IDENTITY VERIFICATION PERFORMANCE ACHIEVED BY THREE DEEP NEURAL NETWORKS FOR THE THREE APPLICATION SCENARIO

\begin{tabular}{lccc}
\hline \hline Method & $\begin{array}{c}\text { PM } \\
\text { EER (\%) }\end{array}$ & $\begin{array}{c}\text { AM } \\
\text { EER (\%) }\end{array}$ & $\begin{array}{c}\text { AM-vs-PM } \\
\text { EER (\%) }\end{array}$ \\
\hline VGG-16 & 5.79 & 17.93 & 30.77 \\
Resnet-50 & 1.50 & 11.87 & 15.21 \\
SENet & 2.06 & 10.36 & 13.21 \\
\hline \hline
\end{tabular}

\section{REFERENCES}

[1] L. Olivieri, D. Mazzarelli, B. Bertoglio, D. Angelis, C. Previderé, P. Grignani, A. Cappella, S. Presciuttini, C. Bertuglia, P. Simone, N. Polizzi, A. Iadicicco, V. Piscitelli, and C. Cattaneo, "Challenges in the identification of dead migrants in the Mediterranean: The case study of the Lampedusa shipwreck of October 3rd 2013," Forensic Science International, vol. 285, February 2018.

[2] Z. Caplova, Z. Obertova, D. M. Gibelli, D. Mazzarelli, T. Fracasso, P. Vanezis, C. Sforza, and C. Cattaneo, "The reliability of facial recognition of deceased persons on photographs," Journal of Forensic Sciences, vol. 62, no. 5, pp. 1286-1291, 2017

[3] A. Anand, R. Donida Labati, A. Genovese, E. Munoz, V. Piuri, and F. Scotti, "Age estimation based on face images and pre-trained convolutional neural networks," in Proc. of the IEEE Symposium Series on Computational Intelligence (SSCI), 2017, pp. 1-7.

[4] D. C. Cornett, D. S. Bolme, D. W. Steadman, K. A. Sauerwein, and T. B. Saul, "Effects of postmortem decomposition on face recognition," in Proc. of the IEEE 10th International Conference on Biometrics Theory, Applications and Systems (BTAS), 2019, pp. 1-8.

[5] P. Majumdar, S. Chhabra, R. Singh, and M. Vatsa, "Subclass contrastive loss for injured face recognition," in Proc. of the IEEE 10th International Conference on Biometrics Theory, Applications and Systems (BTAS), 2019, pp. 1-7.

[6] V. Piscitelli, A. Iadicicco, D. De Angelis, D. Porta, and C. Cattaneo, "Italy's battle to identify dead migrants," The Lancet Global Health, vol. 4, no. 8, pp. e512-e513, 2016.

[7] K. Panetta, S. Rajeev, K. M. S. Kamath, and S. S. Agaian, "Unrolling post-mortem $3 \mathrm{~d}$ fingerprints using mosaicking pressure simulation technique," IEEE Access, vol. 7, 2019.

[8] A. Boyd, S. Yadav, T. Swearingen, A. Kuehlkamp, M. Trokielewicz, E. Benjamin, P. Maciejewicz, D. Chute, A. Ross, P. Flynn, K. Bowyer, and A. Czajka, "Post-mortem iris recognition-a survey and assessment of the state of the art," IEEE Access, vol. 8, pp. 136570-136593, 2020.

[9] P. J. Phillips, A. N. Yates, Y. Hu, C. A. Hahn, E. Noyes, K. Jackson, J. G. Cavazos, G. Jeckeln, R. Ranjan, S. Sankaranarayanan, J.-C. Chen, C. D. Castillo, R. Chellappa, D. White, and A. J. O'Toole, "Face recognition accuracy of forensic examiners, superrecognizers, and face recognition algorithms," Proc. of the National Academy of Sciences, vol. 115, no. 24, pp. 6171-6176, 2018.

[10] S. Nagpal, M. Singh, A. Jain, R. Singh, M. Vatsa, and A. Noore, "On matching skulls to digital face images: A preliminary approach," in Proc. of the IEEE International Joint Conference on Biometrics (IJCB), 2017, pp. 813-819.

[11] M. Taskiran, N. Kahraman, and C. E. Erdem, "Face recognition: Past, present and future (a review)," Digital Signal Processing, vol. 106, 2020.

[12] M. Wang and W. Deng, "Deep face recognition: A survey," Neurocomputing, vol. 429, pp. 215-244, 2021.

[13] R. Donida Labati, A. Genovese, E. Muñoz, V. Piuri, F. Scotti, and G. Sforza, "Computational intelligence for biometric applications: a survey," International Journal of Computing, vol. 15, no. 1, pp. 40-49, 2016.

[14] W. J. Scheirer, A. de Rezende Rocha, A. Sapkota, and T. E. Boult, "Toward open set recognition," IEEE Trans. on Pattern Analysis and Machine Intelligence, vol. 35, no. 7, 2013.

[15] A. Kumar, A. Kaur, and M. Kumar, "Face detection techniques: A review," Artificial Intelligence Review, vol. 52, no. 2, p. 927-948, Aug. 2019.

[16] O. M. Parkhi, A. Vedaldi, and A. Zisserman, "Deep face recognition," in Proc. of the British Machine Vision Conference (BMVC), September 2015, pp. 41.1-41.12.
[17] K. He, X. Zhang, S. Ren, and J. Sun, "Deep residual learning for image recognition," in Proc. of the 2016 IEEE Conf. on Computer Vision and Pattern Recognition (CVPR), 2016, pp. 770-778.

[18] J. Hu, L. Shen, and G. Sun, "Squeeze-and-excitation networks," in Proc. of the 2018 IEEE/CVF Conference on Computer Vision and Pattern Recognition, 2018, pp. 7132-7141.

[19] Y. Guo, L. Zhang, Y. Hu, X. He, and J. Gao, "Ms-celeb-1m: A dataset and benchmark for large-scale face recognition," in Proc. of the European Conference on Computer Vision (ECCV), B. Leibe, J. Matas, N. Sebe, and M. Welling, Eds., 2016, pp. 87-102.

[20] Q. Cao, L. Shen, W. Xie, O. M. Parkhi, and A. Zisserman, "Vggface2: A dataset for recognising faces across pose and age," in Proc. of the 13th IEEE International Conference on Automatic Face Gesture Recognition (FG), 2018, pp. 67-74.

[21] S. Liu and W. Deng, "Very deep convolutional neural network based image classification using small training sample size," in Proc. of the 3rd IAPR Asian Conference on Pattern Recognition (ACPR), 2015, pp. 730-734.

[22] F. Schroff, D. Kalenichenko, and J. Philbin, "Facenet: A unified embedding for face recognition and clustering," in Proc. of the IEEE Conference on Computer Vision and Pattern Recognition (CVPR), 2015, pp. $815-823$.

[23] K. Zhang, Z. Zhang, Z. Li, and Y. Qiao, "Joint face detection and alignment using multitask cascaded convolutional networks," IEEE Signal Processing Letters, vol. 23, no. 10, pp. 1499-1503, 2016.

[24] D. Maio, D. Maltoni, R. Cappelli, J. Wayman, and A. Jain, "FVC2000: Fingerprint verification competition," IEEE Trans. Pattern Anal. Mach. Intell., vol. 24, pp. 402-412, March 2002.

[25] A. K. Jain, P. Flynn, and A. A. Ross, Handbook of Biometrics. New York, NY, USA: Springer Sci. \& Bus. Media, 2007. 